\title{
Evaluation and Measurement of the Wrinkle-Reduction Performance of a Non- Toxin Based Topical Skin Serum on Volunteers
}

\author{
Allanah M Knight ${ }^{1}$ and Sharad P Paul ${ }^{1,2, *}$ \\ ${ }^{1}$ Skin Surgery Clinic, Auckland \\ ${ }^{2}$ Auckland University of Technology, New Zealand
}

*Corresponding author: Paul SP, Skin Surgery Clinic, Adjunct Professor, Auckland University of Technology, New Zealand, E-mail: doctor@ skinsurgeryclinic.co.nz

Received: 30 Nov, 2020 | Accepted: 30 Dec, 2020 | Published: 11 Jan, 2021

Citation: Knight AM, Paul SP (2021) Evaluation and Measurement of the Wrinkle-Reduction Performance of a Non-Toxin Based Topical Skin Serum on Volunteers. J Clin Cosmet Dermatol 4(4): dx.doi.org/10.16966/2576-2826.159

Copyright: (c) 2020 Knight AM, et al. This is an open-access article distributed under the terms of the Creative Commons Attribution License, which permits unrestricted use, distribution, and reproduction in any medium, provided the original author and source are credited.

\section{Abstract}

Objective: The primary aim of this study was to evaluate the performance of a commercially manufactured study product-a non-toxin-based topical skin serum-in terms of its wrinkle reducing capability in women concerned about the appearance of their wrinkles.

Patients and Methods: 21 female volunteers, Fitzpatrick skin types I-III, signed up to participate in this prospective, open-label, within-subject controlled study, which was performed under medical supervision in a single centre. Due to Covid-19 regional lockdowns and travel restrictions, some participants had to withdraw from the study and 13 volunteers completed the study. After an initial visit at baseline to verify adherence to the protocol criteria, volunteers underwent a baseline non-invasive 3D skin scan and received a dermatologically formulated serum devoid of paralytic toxins (No-Tox ${ }^{\circledR}$ ) to begin topical application over wrinkles at a self- nominated facial site of concern (forehead, nasolabial or periorbital) followed 3 weeks later by a second skin scan to monitor progress, and a final scan at 6 weeks. In all 3 visits, the last performed 6 weeks after the first serum application, were conducted to evaluate clinically and instrumentally the efficacy of the treatment.

Results: Clinical and statistically significant reduction in wrinkles was recorded after the first post-treatment visit at 3 weeks, and the effect was maintained progressively until the end of the study period of 6 weeks and 12 weeks. A clinically measurable amelioration of wrinkle severity was also observed. By contrast, untreated areas did not show any improvement. Using 3D imaging and quantitative analysis of these skin scans, it was possible to determine the efficacy which was already appreciable at the first visit at 3 weeks, and further improvement was noticed at 6 weeks and beyond. The serum was very well tolerated by the volunteers, as evidenced by the absence of adverse reactions or skin sensitivities.

Conclusion: The results of the study confirm the significant aesthetic improvements of the non-toxin-based serum ( No-Tox ${ }^{\circledR}$ ) in reducing wrinkle volume and the appearance of fine lines. The wrinkle reducing activity of the study product was demonstrable on 3D skin imaging. The limitations of the study are the small numbers of participants, and that the participants were of the Fitzpatrick type I-III skin types only. However, the results indicate that the serum has the potential in other skin types, and further studies are planned.

Keywords: Wrinkles; Aging; Actinic damage; Clinical assessment; Instrumental assessment; Cosmetics

\section{Introduction}

If we consider human skin as a mere physical membrane, wrinkles fundamentally occur because a keratinocyte-stiffened epidermis drapes a softer and thicker dermis [1]. Skin aging-either extrinsic mainly due to ultraviolet (UV) damage, or intrinsic due to physiological factors-is characterized by features such as wrinkling, loss of elasticity, and rough-textured appearance of skin [2]. Around 80 percent of skin aging is because of sun damage [3], and in places like New Zealand and Australia, or in the Sunbelt of America such as Florida and California, this is especially noticeable at younger ages. For women, a significant concern is the appearance of wrinkles and considerable expenses are incurred on anti-aging pharmaceuticals and cosmetics
[4]. There have been several studies evaluating different modalities of wrinkle-reduction. There is no doubt that invasive methods that use neurotoxins to paralyse muscles can eliminate dynamic wrinkles such as horizontal forehead lines instantly. Studies have confirmed the efficacy of injecting paralytic toxins such as Botulinum toxin Type A in the management of dynamic forehead wrinkles caused by muscle action, with affects that persist for up to 16 weeks [5]. Others have reviewed the efficacy of injected intradermal fillers such as nonpermanent skin fillers using collagen-based materials and hyaluronic acid, or semi-permanent fillers including poly methyl methacrylate, poly L-lactic acid and calcium hydroxyapatite microspheres, or permanent fillers including silicone that have been used in attempts 
to reduce wrinkles and lines [6]. Non pharmaceutical cosmetic facial serums in combination with washes and moisturizers have also been studied on photodamaged skin and may improve the appearance of fine lines and wrinkles [7]. Our aim was to test this commercially manufactured serum containing plant and plankton extracts, and peptides, and free of paralytic toxins-to evaluate this skin serum's ability to rapidly reduce wrinkles and lines.

No-Tox ${ }^{\oplus}$ (No-Tox Limited, New Zealand), is a topical serum that contains Plukenetia volubilis seed extracts, plankton extracts, glucosamine, hyaluronic acid, niacinamide and copper blended with other ingredients. The serum is devoid of any paralytic toxins. Many of the ingredients in this commercially manufactured proprietary blend are individually known to have beneficial properties on skin and increase elastin and collagen production. For example Plukenetia volubilis oil has been shown to be a potent moisturizer with low irritant potential [8]; Glucosamine has shown to improve skin hydration, wrinkle reduction, and help control skin pigmentation disorders when used both topically and orally [9]. Almost all hyaluronic acidbased skin products have demonstrated a significant improvement in skin hydration and overall elasticity values, and therefore have the potential to reduce skin wrinkling [10]. However with hyaluronic acid, the relationship is not linear and therefore quantities used in formulations are important. For example, wrinkly dogs like Shar Peis have mucinosis -with high hyaluronic acid levels in both cutaneous tissues and the blood stream- due to an excess in the activity (overexpression) of the HAS2 enzyme that produces more hyaluronic acid [11]. Niacinamide in topical preparations has been shown to cause an improved appearance of skin wrinkles, reducing yellowing and improving skin elasticity [12]. Tocopherol (vitamin E), when applied topically to skin has been shown to reduce erythema after sunburn, chronic UVB-induced skin damage, and carcinogenesis in several published studies $[13,14]$. Copper along with peptide complexes have been reported to increase collagen levels in the skin, in studies that have used immunohistochemistry [15].

The product was supplied to our skin clinic-based research lab to be evaluated independently for its performance and duration of effect as a potential wrinkle-reducing topical agent in volunteer women. The study was approved by an independent ethics committee and conducted free of financial inducements. No remuneration was received for the conduct of this study by either researchers or participants. The research objective was simply to assess if this product was beneficial in reducing wrinkles, or not. The product is commercially manufactured at a facility in Chatsworth, CA, USA (FDA Registration FEI No. 3006402197).

\section{Subjects and Methods}

The study was planned as a prospective, open-label, within-subject controlled, single-centre study conducted on 21 healthy female subjects under dermatological control. The aim was a 12 -week, singlearm, open-label controlled trial performed at a single site in New Zealand with evaluations at $0,3,6$ and 12 weeks after commencing treatment. Subjects and investigators were not blinded to the treatment serum, which was provided in its commercial packaging. But because of Covid-19, the duration of the study was reduced, and only 13 participants were able to attend for 6 weeks. Two participants were available for a final scan at 12 weeks.

Subjects were required to be female volunteers, between the ages of 30-75 and with concerns regarding facial wrinkling. The mean age of the subjects ended up 54 years (range 41-72 years), and of Fitzpatrick skin types I to III, with early to advanced photo damaged facial skin. There were no specific exclusions in skin type other than the ability to provide informed consent, and subjects could continue to use their normal cosmetic regimen. They were instructed to use the study serum once daily at night after cleansing skin and 2-4 drops of serum was smoothed into the specific area of wrinkles being treated. In general, participants applied the serum to one of three areas chosen by themselves to be their main area of cosmetic concern - forehead/ glabellar region, upper lip (cutaneous labial and nasolabial region) and eye region (crow's feet or under-eye region)-with respect to both fine lines and coarse wrinkles (Figure 1).

The study was performed according to the guidelines of the Declaration of Helsinki with respect to ethical principles for medical research involving human subjects. All the participants gave their informed and voluntary consent after reading the study documents for participation as well as for the publication of any or all of their images and photographs. Over 30 days were allowed from the invitation to the effective study participation date, to fulfill the Declaration of Helsinki principles.

\section{Clinical assessment}

Digital photographs were taken and subjects' skin condition was assessed in terms of facial lines and wrinkles, the suitability of the sites chosen for topical application, and also any skin reactions or allergies were noted at each visit. Signs and symptoms of irritation were evaluated both clinically (erythema, dryness, and peeling) and subjectively (burning, stinging, itching or tingling) using a 4-point scale (where $0=$ none, $1=$ mild, $2=$ moderate, and $3=$ severe).

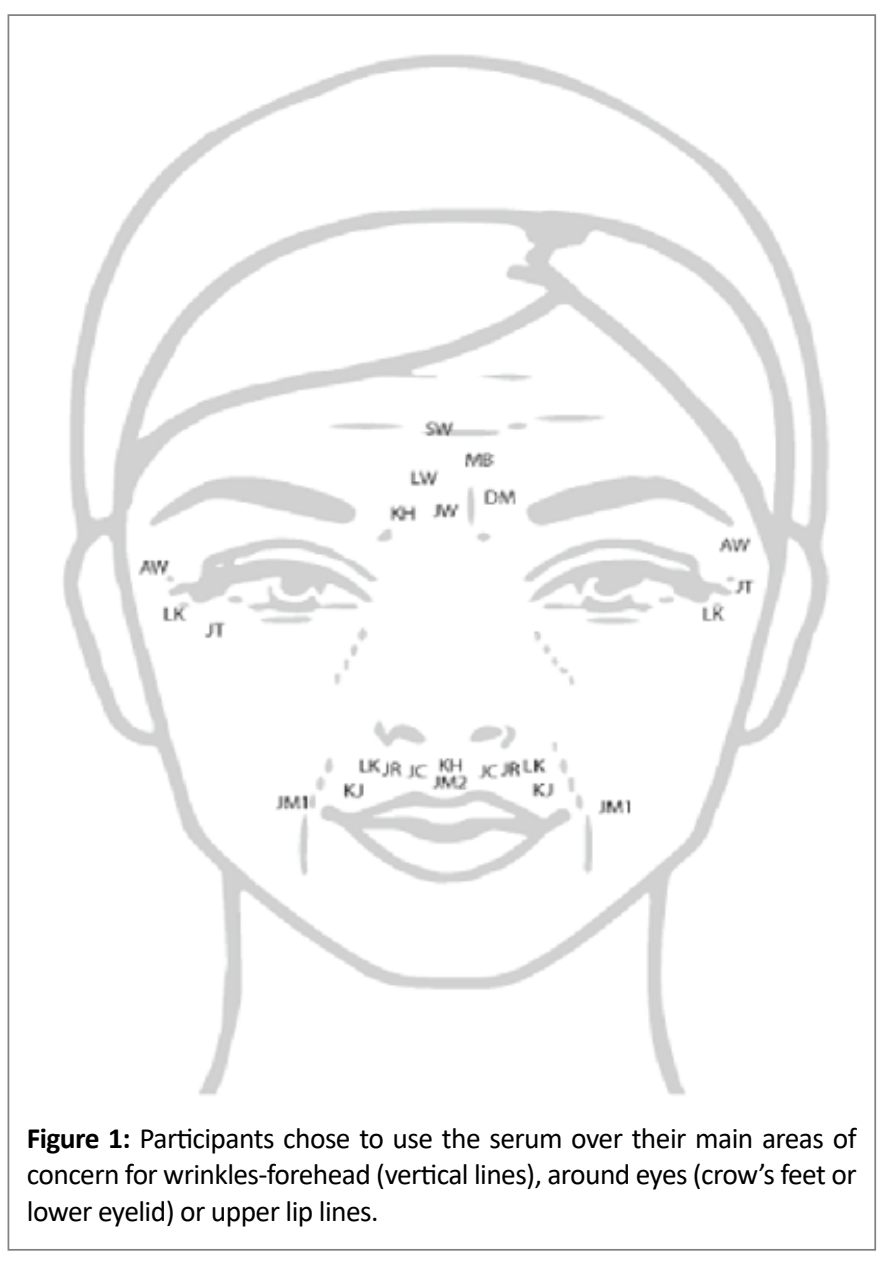




\section{Quantitative instrument measurement}

Wrinkle measurement can be undertaken directly using a clinical score and instruments such as surface photometry, or indirectly using skin wrinkle replication technology and computer software analysis that embedded into several skin scanners. Measurements of wrinkles and percentage changes in wrinkles over the duration of this study were measured using the Pear 3D (DermaQuip LLC, Marietta, GA 30062, USA) scanner.

In recent times, wrinkle and lines have been studied non-invasively by using a variety of $3 \mathrm{D}$ skin scanners. These scanners use a multitude of light sources-standard incandescent light, UV light and polarized light. The standard flash light can identify spots, wrinkle, skin texture, and pores while the ultraviolet flash light is used to analyse the ultraviolet damage and porphyrin. Cross-polarized flash light is helpful for observing brown spots and red areas.

The Pear 3D system used in this study utilizes three light sourcesnormal, polarized, and Ultraviolet (UV). Autofocus is achieved at a resolution of 15 million auto pixels. Normal light is used for spot, wrinkle, texture and pore analysis. UV light is for assessment of sundamage below the surface, as well as bacterial counts on skin. The polarized light is used for the surface sun damage as well as to analyse blood vessels on the skin.

The research team is experienced in the use and technology of skin scanners. VISIA (Canfield Imaging Systems, NJ, USA) is similar to the Pear 3D but has the added RBX technology that is useful to analyse brown and red spots [16]. The ANTERA 3D (Miravex Limited) is another device which contains a camera for image acquisition and corresponding software for analysis of the skin. Results are derived from the spatial and spectral analysis of the acquired image data, obtained by illuminating the skin with LEDs of different wavelengths from different directions. The ANTERA 3D uses a computer to analyse the differences between these images and reconstruct the surface in $2 \mathrm{D}$ and 3D [16]. The Pear 3D system chosen for this study was easy to use, results reproducible in the same person, and the ability of the Pear 3D scanner to focus on target areas to assess wrinkles in $3 \mathrm{D}$ was especially useful to this research team. Changes in wrinkle percentage were indicated as a percentage indicating either an increase or decrease in wrinkle visibility. In general, wrinkles less than $1 \mathrm{~mm}$ in width and depth are defined as fine lines or wrinkles, and wrinkles that are 1 $\mathrm{mm}$ or more in width and depth are defined as coarse wrinkles [17]. The changes noted in wrinkle volume are essentially an average of the changes in both fine lines and coarse wrinkles.

\section{Statistical analysis}

Instrument measurements were undertaken for changes in wrinkles at the three selected sites (forehead, lip and eye regions) at 3 weeks, 6 weeks and 12 weeks compared to baseline. The data was independently analysed by the mathematical sciences and statistics department of the Auckland University of Technology. Linear regression models and unpaired t-tests were used to analyse the relationship between the changes to wrinkles and variables such as age and treatment sites, and the statistical differences in wrinkle volumes between treated and untreated sites. The results are plotted in figures 2 and 3.

\section{Results}

Originally, the study was devised with 21 participants over a 12 week period but the final number was lower due to matters unrelated to the study. Due to Covid-19 regional lockdown restrictions imposed by the New Zealand government, 13 participants were able to attend for their scans at 0,3 and 6 weeks only, and therefore the study duration was shortened for 6 weeks, as a second government lockdown was imposed at this point in time. Quantitative instrument and data analysis was, therefore, performed on a total of 13 patients up to 6 weeks, and 2 patients up to 12 weeks. Analysis was performed at treated and untreated sites-for example if a person had chosen to apply the study serum on the forehead region, mean changes in wrinkles were also analysed at the untreated sites to provide a within participant control arm. One participant was removed from the final analysis as the serum had been applied to multiple sites on the face, and this had the potential to affect the control arm.

Linear regression models were created in $\mathrm{R}$. The null hypothesis was that there was no significant $p$ value for a null i.e. zero effectiveness when split between treated and untreated sites, or by location.

There was statistically a very significant reduction in wrinkle volume between treated and untreated sites ( $p$ value: $2.997 \mathrm{e}-05)$ at all facial locations (Table 1 ). When compared by specific locations on the face, at 0-3 weeks the efficacy was not statistically different between different regions, but at 3-6 weeks, there was a difference noted--with eyelid and upper lip areas showing greater reduction of wrinkles/lines than the forehead (Figure 3). The age of participants did not appear to influence efficacy either at 0-3 weeks or at 3-6 weeks ( $\mathrm{p}$ value: 0.4551) (Figure 2). All facial regions showed statistically significant improvements when compared with untreated areas.

\section{Efficacy}

The efficacy of the study serum in causing progressive reduction of coarse wrinkles and fine lines in the areas where the product was used could be demonstrated by the investigator (with clinical photographs (Figure 4)), further confirmed by instrument analysis (using the Pear 3D scanner (Figure 5)), and finally corroborated by statistical results (Figure 6).
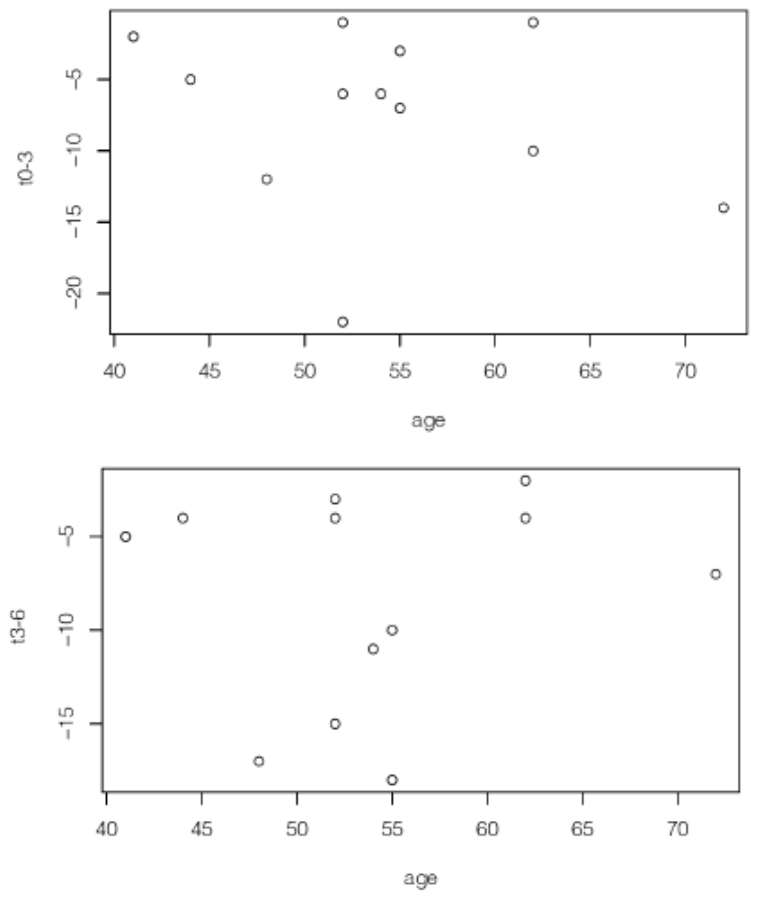

Figure 2: Wrinkle reduction was noted in all participants, and age did not appear to make a significant difference either at 0-3 or 3-6 week periods. 

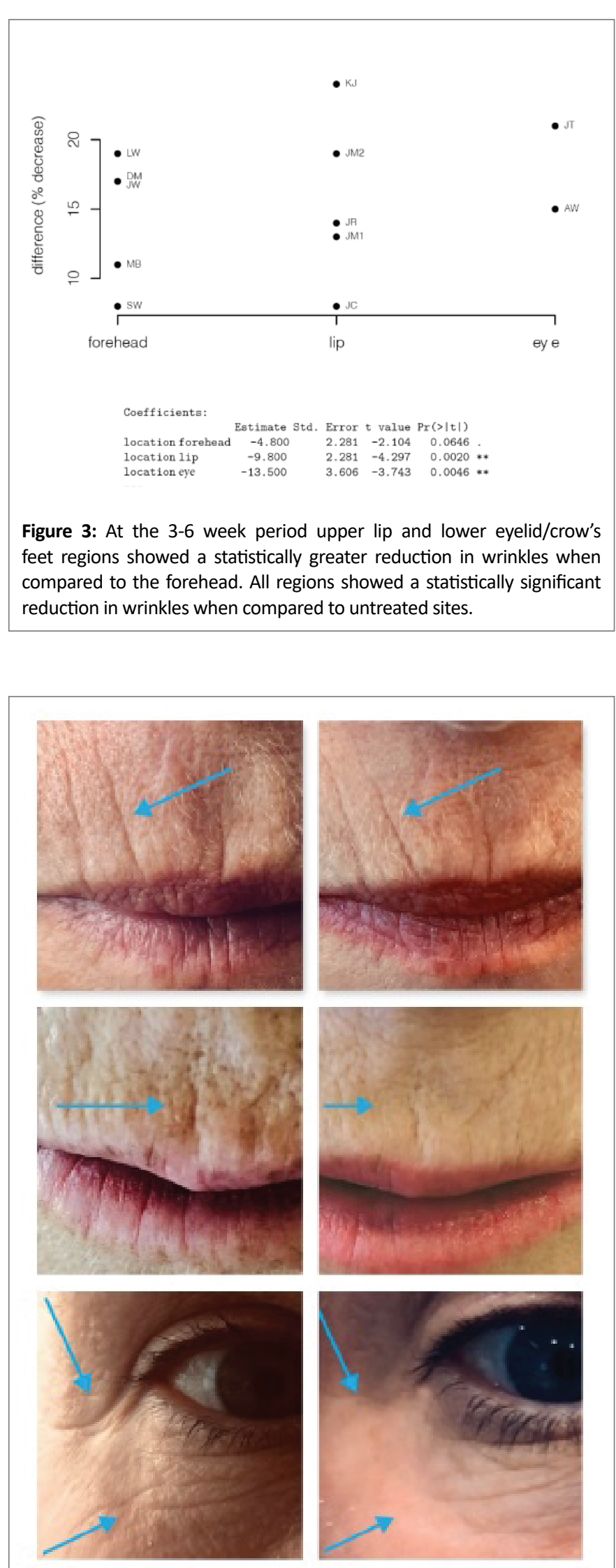

Figure 4: Examples of before and after clinical photographs that indicate reduction in fine and coarse wrinkles.

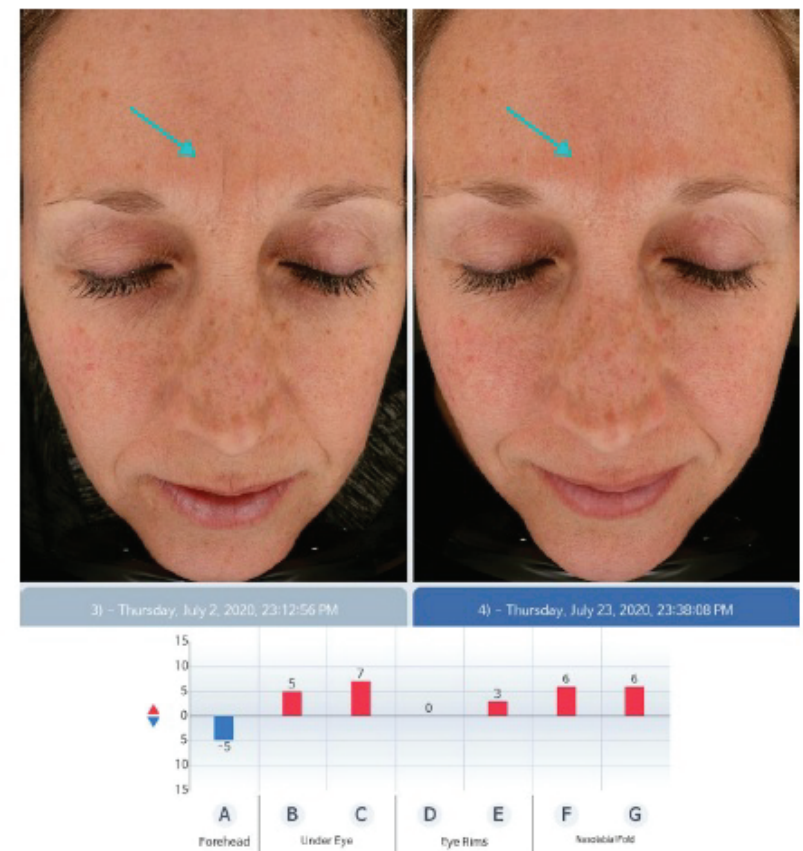

Figure 5: Representative images from the facial scans that demonstrate the reduction of forehead wrinkles at 3 weeks; importantly untreated areas did not show any reduction.

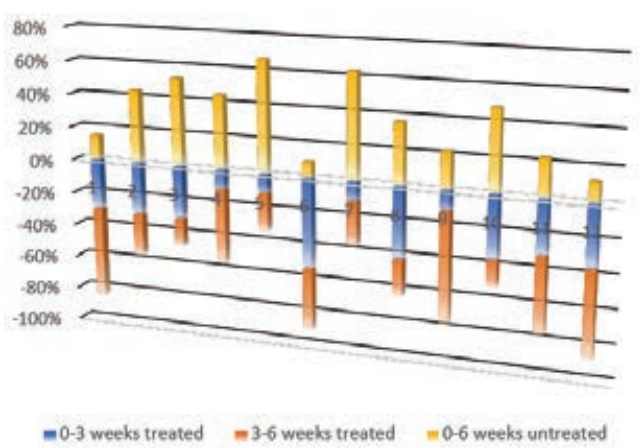

Figure 6: Percentage change in coarse wrinkles and fine lines over the study period.

The facial serum showed statistically significant within-group improvement in the appearance of fine lines and wrinkles over the study period. Significant improvements $(P \leq 0.05)$ in quantitative measurements i.e. reduction of wrinkle depth percentage were seen as early as Week 3 , progressively improved at Week 6 and were maintained at Week 12. Clinically changes were noted in both coarse and fine wrinkles, although the changes were more marked in the latter.

By Week 3, participant scans revealed a mean $7 \%$ decrease in visible wrinkles (range $1 \%$ to $22 \%$ ). By Week 6, facial scans showed the reduction in wrinkles further reduced from the Week 3 mark by another $8 \%$ (range $2 \%$ to $18 \%$ ). In contrast, untreated areas revealed a mild increase in wrinkles, further illustrating the photo damage caused by the harsh southern hemisphere sun in New Zealand especially in fair-skinned individuals. Participants that had greater sun exposure 
Table 1: Percentages changes in wrinkle volume at 0-3 weeks, 3-6 weeks and 6-12 weeks at treated sites, and 0-6 weeks at untreated sites ( $t=$ time).

\begin{tabular}{|c|c|c|c|c|c|c|c|}
\hline & Age & $\mathbf{t 0 - 3}$ & $\mathbf{t 3 - 6}$ & $\mathbf{t 6 - 1 2}$ & $\mathbf{u 0 - 6}$ & initials & Location \\
\hline $\mathbf{1}$ & 54 & -6 & -11 & NA & 3 & JR & Lip \\
\hline $\mathbf{2}$ & 44 & -5 & -4 & NA & 7 & MB & Forehead \\
\hline $\mathbf{3}$ & 52 & -6 & -3 & NA & 10 & JM1 & Lip \\
\hline $\mathbf{4}$ & 52 & -1 & -4 & NA & 4 & SW & Forehead \\
\hline $\mathbf{5}$ & 62 & -1 & -2 & -2 & 6 & JC & Lip \\
\hline $\mathbf{6}$ & 52 & -22 & -15 & NA & 4 & JM2 & Lip \\
\hline $\mathbf{7}$ & 41 & -2 & -5 & -4 & 12 & JW & Forehead \\
\hline $\mathbf{8}$ & 72 & -14 & -7 & NA & 12 & LW & Forehead \\
\hline $\mathbf{9}$ & 55 & -3 & -18 & NA & 6 & KJ & Lip \\
\hline $\mathbf{1 0}$ & 62 & -10 & -4 & NA & 13 & DM & Forehead \\
\hline $\mathbf{1 1}$ & 55 & -7 & -10 & NA & 5 & AW & Eye Region \\
\hline $\mathbf{1 2}$ & 48 & -12 & -17 & NA & 4 & JT & Eye Region \\
\hline
\end{tabular}

showed a more marked deterioration in untreated areas. 11 of the 13 participants $(84.2 \%)$ felt that their fine lines and coarse wrinkles were visibly reduced in the areas treated, and signs of aging had reduced significantly by Week 6 .

\section{Tolerability}

Overall, the study serum was exceptionally well tolerated. Skin irritation, erythema, dryness, redness or stinging was not reported by any participant at any stage of the study, even by participants using it in the eyelid areas.

\section{Conclusion}

The results of the study confirm the significant aesthetic improvements when using the study serum in coarse wrinkle and fine line reduction. The wrinkle reducing activity of the study serum was maintained progressively over 3 and 6 weeks, and beyond. These effects were obtained with no undesired effects or skin reactions. Statistically significant improvements were seen both clinically and using the quantitative study parameters as early as 3 weeks.

Subject satisfaction and cutaneous tolerability were excellent. Overall, this study suggests that this non-toxin-based skin serum (No$\left.\mathrm{Tox}^{\oplus}\right)$ is effective in reducing wrinkles and lines. The study is limited by the small sample size, although the within-participant control arm, and statistical results achieved do show very significant $p$ values. Another limitation is that the participants were limited to Fitzpatrick I-III skin types, not by design, but due to availability during the study period, and the predominant local population. The results do not suggest that the serum may not be effective in other skin types. More formal clinical trials with participants with other skin types, including skin biopsy studies (to understand collagen and elastin changes) are planned in the future to augment the findings of this study.

\section{Acknowledgments}

The authors gratefully acknowledge the volunteers for their participation in this study. The authors are especially grateful to Robin Hankin of the Mathematical Sciences Department, Auckland University of Technology for undertaking independent statistical analyses of the data.

\section{Disclosure}

AK has no conflict of interest to declare. SP is a shareholder of NoTox (NZ) Limited. No fees or grants have been paid or authorised to any party for undertaking this study. Both AK and SP are employees of the Skin Surgery Clinic.

\section{References}

1. Paul SP (2016) The Epistemology of Wrinkles: From Geology and Anatomy to Physiology. Int J Biomed 6: 237-239.

2. Zhang S, Duan E (2018) Fighting against Skin Aging: The Way from Bench to Bedside. Cell Transplant 27: 729-738.

3. Friedman $O(2005)$ Changes associated with the aging face. Facial Plast Surg Clin North Am 13: 371-380.

4. Kazanci A, Kurus M, Atasever A (2017) Analyses of changes on skin by aging. Skin Res Technol 23: 48-60.

5. Susmita A, Kolli NND, Meka S, Chakravarthi SP, Kattimani VS, et al. (2016) An Evaluation of Use of Botulinum Toxin Type $A$ in the Management of Dynamic Forehead Wrinkles - A Clinical Study. J Clin Diagn Res 10: ZC127-ZC131.

6. Lin ZYW, Shah V, Dhinakar A, Yildirimer L, Cui WG, et al. (2016) Intradermal fillers for minimally invasive treatment of facial aging. Plast Aesthet Res 3: 72-82.

7. McCall-Perez F, Stephens TJ, Herndon JH Jr. (2011) Efficacy and tolerability of a facial serum for fine lines, wrinkles, and photodamaged skin. J Clin Aesthet Dermatol 4: 51-54.

8. Soimee W, Nakyai W, Charoensit P, Grandmottet F, Worasakwutiphong $\mathrm{S}$, et al. (2020) Evaluation of moisturizing and irritation potential of sacha inchi oil. J Cosmet Dermatol 19: 915-924.

9. Bissett DL (2006) Glucosamine: an ingredient with skin and other benefits. J Cosmet Dermatol 5: 309-315.

10. Pavicic T, Gauglitz GG, Lersch P, Schwach-Abdellaoui K, Malle B, et al. Efficacy of cream-based novel formulations of hyaluronic acid of different molecular weights in anti-wrinkle treatment. J Drugs Dermatol 10: 990-1000.

11. Olsson M, Meadows JRS, Truve K, Pielberg GR, Puppo F, et al. (2011) A Novel Unstable Duplication Upstream of HAS2 Predisposes to a Breed-Defining Skin Phenotype and a Periodic Fever Syndrome in Chinese Shar-Pei Dogs. PLoS Genet 7: e1001332.

12. Bissett DL, Oblong JE, Berge CA (2005) Niacinamide: A B vitamin that improves aging facial skin appearance. Dermatol Surg 31: 860-865.

13. McVean M, Liebler DC (1999) Prevention of DNA photodamage by vitamin $\mathrm{E}$ compounds and sunscreens: roles of ultraviolet absorbance and cellular uptake. Mol Carcinog 24: 169-176.

14. Schagen SK, Zampeli VA, Makrantonaki E, Zouboulis CC (2012) Discovering the link between nutrition and skin aging. Dermatoendocrinol 4: 298-307.

15. Abdulghani A, Sherr A, Shirin S, Solodkina G, Tapia E, et al. (1998) Effects of topical creams containing vitamin $\mathrm{C}$, a copper-binding peptide cream and melatonin compared with tretinoin on the ultrastructure of normal skin - a pilot clinical, histologic, and ultrastructural study. Dis Manag Clin Outcomes 1: 136-141.

16. Linming F, Wei H, Anqi L, Yuanyu C, Heng X, et al. (2018) Comparison of two skin imaging analysis instruments: The VISIA ${ }^{\circledR}$ from Canfield vs the ANTERA 3D ${ }^{\circledR}$ CS from Miravex. Skin Res Technol 24: 3-8.

17. Manriquez JJ, Cataldo K, Vera-Kellet C, Harz-Fresno I (2014) Wrinkles. BMJ Clin Evid 1711. 